\title{
Mesurer et décrire : savoir géographique et cartographie dans l'espace germanique protestant (des années 1530 aux années 1620)
}

\section{Axelle Chassagnette}

\section{OpenEdition}

\section{Journals}

Édition électronique

URL : http://journals.openedition.org/ifha/251

DOI : 10.4000/ifha.251

ISSN : 2198-8943

\section{Éditeur}

IFRA - Institut franco-allemand (sciences historiques et sociales)

\section{Édition imprimée}

Date de publication : 1 janvier 2010

Pagination : 194-200

ISSN : 2190-0078

\section{Référence électronique}

Axelle Chassagnette, « Mesurer et décrire : savoir géographique et cartographie dans l'espace germanique protestant (des années 1530 aux années 1620) », Revue de l'IFHA [En ligne], 2 | 2010, mis en ligne le 01 février 2013, consulté le 20 avril 2019. URL : http://journals.openedition.org/ifha/251 ; DOI : 10.4000/ifha. 251

Ce document a été généré automatiquement le 20 avril 2019.

(CIFHA 


\title{
Mesurer et décrire : savoir géographique et cartographie dans l'espace germanique protestant (des années 1530 aux années 1620)
}

\author{
Axelle Chassagnette
}

\section{NOTE DE L'ÉDITEUR}

Axelle Chassagnette, Mesurer et décrire : savoir géographique et cartographie dans l'espace germanique protestant (des années 1530 aux années 1620), Thèse pour le doctorat en histoire sous la direction de Gérald Chaix (Université de Tours, CESR), soutenue le 28 novembre 2009. Axelle Chassagnette a été de 2007 à 2009 boursière de la MHFA de Göttingen et bénéficiaire en 2009-2010 d'une aide à la mobilité de l'IFHA. Elle est actuellement professeur d'histoire-géographie en section Abibac au lycée Jean-Monnet de Strasbourg.

1 Quand et comment la géographie est-elle née ? Quels processus ont conduit ce domaine de savoir à se constituer en discipline ? Ces questions sont à la source du travail de thèse présenté ici. Par discipline, nous entendons, d'après une définition empruntée à l'historien Donald Kelley, un domaine de connaissance dont l'homogénéité est culturellement construite, caractérisé par la reconnaissance d'un corpus commun d'autorités, d'un lexique défini, de méthodes intellectuelles et techniques établies, d'un ensemble de problèmes, de questions théoriques et pratiques et de domaines d'application, par l'existence d'une communauté de praticiens et - dans certains cas - par des signes de professionnalisation ${ }^{1}$.

2 Le choix du XVI siècle (plus largement, de la période de la Renaissance) pour mener cette recherche s'explique par les transformations du savoir du monde dans l'Europe de la première modernité. La connaissance de la terre s'élargit en effet au temps des grandes découvertes et des voyages d'exploration vers l'Asie et l'Amérique. La redécouverte, au 
début du $\mathrm{XV}^{\mathrm{e}}$ siècle, du texte de la Géographie du mathématicien et astronome alexandrin Claude Ptolémée permet par ailleurs aux lettrés de s'approprier progressivement un vocabulaire spécifique propre à la description géographique (à la fois graphique et textuelle), des listes de toponymes dont sont données les coordonnées géographiques, ainsi que des techniques de projection et de construction cartographiques ${ }^{2}$. La géographie fait partie des domaines de connaissance qui s'épanouissent au XVI ${ }^{\mathrm{e}}$ siècle et contribuent au déplacement des catégories classificatoires de la connaissance. Entre mathématiques et histoire, description et calcul, elle constitue un objet privilégié pour l'étude des formes du savoir propres à la période de la Renaissance et permet d'interroger les critères de définition et de validation du paradigme historiographique de la Révolution scientifique ${ }^{3}$.

Pourquoi par ailleurs choisir de limiter l'étude à l'espace germanique? Les princes du Saint-Empire n'ont certes guère participé aux entreprises maritimes des Grandes Découvertes. Les lettrés et humanistes allemands ont en revanche contribué avec enthousiasme à la réception et la diffusion des nouvelles connaissances géographiques. Ce fait s'explique notamment par un intérêt précoce des savants allemands (en particulier Regiomontanus et Peuerbach) pour les mathématiques, l'astronomie et la géographie ${ }^{4}$. Au tournant $\mathrm{du} \mathrm{XV}^{\mathrm{e}}$ siècle, les humanistes allemands manifestent par ailleurs le désir d'établir un nouveau portrait de l'Allemagne, qui présenterait leur patrie sous un jour plus favorable que ne l'ont fait les descriptions de l'Antiquité classique - en particulier la Germania de Tacite. Le projet d'une description géographique de l'Allemagne est proposé par Conrad Celtis, par ailleurs promoteur d'un programme pédagogique humaniste, attentif en particulier à l'enseignement de l'histoire et des mathématiques. Resté inachevé par Celtis, ce projet est poursuivi par de nombreux lettrés du Saint-Empire et des cantons suisses au XVI siècle: les descriptions géographiques et les cartes de l'Allemagne et de ses parties se multiplient et sont imprimées en nombre. La Cosmographie (1544) de Sebastian Münster, dont la plus longue partie est consacrée au Teutschland et dont les nombreuses rééditions en font un des livres les plus lus dans l'Europe de la Renaissance, en est un exemple célèbre ${ }^{5}$.

Pourquoi enfin les territoires protestants? Cette limitation résulte d'un double constat. Le premier, purement pragmatique, est celui de la nécessaire sélection du corpus de sources étudié, d'autant plus nécessaire que l'étude est menée sur une période assez longue (un quasi-siècle). Le second constat est historiographique et découle de quelques études consacrées aux milieux protestants allemands de la première modernité et à l'intérêt que portent les savants issus de ces milieux aux savoirs mathématiques et à l'étude de la nature ${ }^{6}$. Philipp Melanchthon, collaborateur de Martin Luther et premier réformateur du système éducatif des territoires luthériens, légitime ainsi la philosophie naturelle comme un moyen de consolider la nouvelle doctrine religieuse par la connaissance et la reconnaissance de l'action de la Providence dans le monde. Le savoir géographique et la cartographie, définis comme des savoirs mathématiques, trouvent ainsi une place non négligeable dans l'enseignement de la faculté de philosophie de Wittenberg et d'autres universités protestantes du Saint-Empire: nombre de cartographes et de géographes allemands du $\mathrm{XVI}^{\mathrm{e}}$ siècle ont acquis tout ou partie de leur intérêt et de leur formation dans ces établissements ${ }^{7}$.

5 S'ajoute enfin à ces particularités des milieux savants et protestants allemands le développement des processus de confessionnalisation, à l'œuvre dans le Saint-Empire après la paix d'Augsbourg de 1555. Dans la tradition historiographique allemande (en particulier celle qu'ont développée Heinz Schilling et Wolfgang Reinhard $\left.{ }^{8}\right)$, le concept de 
Konfessionalisierung revêt la fonction d'un paradigme explicatif: l'autonomie croissante des princes territoriaux et des conseils urbains dans le domaine des politiques ecclésiales et scolaires est comprise comme un facteur de modernisation des États et de leur administration. Elle est aussi le catalyseur du développement de la perception politique propre des territoires, susceptible de s'exprimer dans le recours croissant à l'inventaire géographique et à la cartographie.

Ces constats apportent un ensemble d'indices convergents qui peuvent permettre de considérer les territoires protestants du Saint-Empire comme des espaces a priori favorables à l'étude du développement du savoir géographique. La limitation de l'étude aux milieux protestants conduit à s'interroger sur la nature des relations qui peuvent lier confession et fabrication de la connaissance, et sur l'incidence des contenus doctrinaux protestants sur le savoir géographique. Cette interrogation s'inscrit plus largement dans un questionnement ancien de l'histoire des sciences, qui, depuis l'ouvrage de Robert Merton sur les puritains anglais au XVII ${ }^{e}$ siècle ${ }^{9}$, a cherché à apprécier l'incidence des positions doctrinales confessionnelles sur la légitimation des savoirs et leur mise en œuvre. Une vulgate "mertonienne", développée sans grande considération pour l'approche relativement prudente et nuancée du sociologue, a voulu faire du protestantisme (à l'opposé du catholicisme) un des facteurs de l'époque moderne favorable à la Révolution scientifique ${ }^{10}$. À l'inverse, notre étude a délibérément voulu se placer en dehors de ce débat et de toute tentative de palmarès. Notre question était plutôt d'apprécier les particularités de la définition et de la pratique du savoir géographique dans l'espace germanique protestant au $\mathrm{XVI}^{\mathrm{e}}$ siècle, compris comme un prisme pour l'étude du développement de ce domaine de connaissance dans l'Europe de la Renaissance. En ce sens, ce travail cherche à construire une étude systématique et emblématique de son objet en même temps qu'elle entend identifier les particularités des pratiques géographiques dans les territoires protestants de l'Empire.

7 La recherche menée tente d'embrasser les sources les plus nombreuses et les plus diversifiées possible sur une période d'un quasi-siècle et dans différents territoires, pour établir un portrait nourri d'un domaine de savoir en plein essor et multiplier les échelles d'analyse. L'approche privilégie les documents qui permettent d'identifier la réalité commune des définitions et des pratiques de la géographie par ses praticiens plutôt que les grandes œuvres imprimées qui ont connu de larges succès de librairie et ont par ailleurs été plus fréquemment étudiées ${ }^{11}$. Il s'agit de manuels et de notes d'enseignement manuscrits et imprimés, d'ouvrages de philosophie et d'encyclopédies, de correspondances savantes, de descriptions géographiques textuelles et de cartes, de journaux d'arpentage et de voyage, d'annonces de cours, de statuts universitaires et autres documents administratifs. Les traités théoriques, philosophiques, pédagogiques et savants portant en partie ou en totalité sur le savoir géographique sont les sources les plus nombreuses et les plus facilement accessibles: elles ont constitué la première strate du corpus documentaire étudié et ont permis d'analyser le statut et les définitions de la géographie dans une perspective désignée comme "internaliste " dans l'histoire des sciences. La recherche s'est ensuite déplacée vers la question de l'enseignement de la géographie, qui nous semblait fondamentale en raison de son importance potentielle dans les processus d'institutionnalisation et d'autonomisation du savoir géographique au regard d'autres domaines de connaissance. Ce travail, complété par un recensement systématique des écrits géographiques et des travaux de cartographie et d'arpentage menés dans différents territoires protestants de l'Empire, a permis de constituer une base 
prosopographique des personnages actifs dans le domaine de la géographie et d'élargir l'étude aux contextes sociaux et politiques de sa mise en œuvre. L'analyse du contenu informatif, formel et matériel des documents produits a par ailleurs permis de mettre en évidence certains aspects des modalités de production et des usages de la géographie.

L'ensemble des analyses est conduit en quatre temps. Le premier traite de l'évolution de la définition théorique et de la classification du savoir géographique au sein de la philosophie, et du statut de ses praticiens. Il accorde une attention particulière aux arguments intellectuels et théologiques qui contribuent à sa légitimation dans les milieux protestants de l'Empire. Le second temps est consacré à la question de l'enseignement géographique dans les universités et les écoles protestantes, et permet de mettre en évidence la formation que reçoivent certains des praticiens de la géographie. Le troisième temps étudie les étapes de la fabrication intellectuelle et matérielle du savoir géographique, s'arrêtant en particulier à l'étude des contextes économiques et politiques de sa production, aux modalités techniques de sa mise en œuvre, aux formes scripturaires, littéraires et graphiques des œuvres textuelles et cartographiques. Enfin, le dernier temps s'interroge sur l'existence d'une spécificité des usages de la géographie dans les milieux protestants allemands, à la faveur d'une part des descriptions de l'espace germanique et de ses territoires, d'autre part des productions de la geographia sacra, cartes et descriptions textuelles destinées à éclairer le texte de l'Écriture et de l'Histoire saintes.

9 L'étude aboutit à deux conclusions principales. La première est que le savoir géographique ne peut qu'en partie être considéré, dans les premières décennies du XVII siècle, comme une discipline à part entière. Domaine de connaissance enseigné de manière régulière dans les universités protestantes du Saint-Empire, disposant d'un corpus d'autorités et d'un vocabulaire propre, la géographie ne constitue cependant jamais l'activité principale de ses praticiens, auxquels elle n'offre pas de statut professionnel. La chronologie de la naissance et de l'essor d'une discipline géographique doit être étendue vers le XVII ${ }^{e}$ siècle. Cette recherche conclut par ailleurs à l'absence d'une "géographie protestante» proprement dite. Si les positions confessionnelles protestantes (elles-mêmes plurielles) contribuent à définir le savoir géographique et à le mettre en œuvre d'une façon parfois particulière et individualisable (ainsi la tendance à «mathématiser » le savoir géographique dans l'entourage de Philipp Melanchthon ou les formes très caractéristiques que revêt la géographie sacrée dans les milieux luthériens et calvinistes), elles insèrent cependant ces définitions et ces usages dans une compréhension du savoir géographique largement partagée par les milieux savants, politiques et administratifs de l'Europe de la première modernité. Certaines de ces positions doctrinales ont cependant permis un essor précoce du savoir géographique dans les territoires protestants de l'Empire. Une étude parallèle et comparative, menée dans les milieux catholiques, devra permettre d'évaluer la spécificité confessionnelle - ou pas de la faveur portée en Allemagne à la connaissance du monde dès le tournant $d u X V^{e}$ siècle. 


\section{NOTES}

1. Donald KeLley (dir.), History and the Disciplines. The Reclassification of Knowledge in Early Modern Europe, Rochester: University of Rochester Press, 1997, introduction.

2. La thèse de la « redécouverte » complète a été nuancée récemment par Patrick GAUTIER-DALCHÉ. Voir La géographie de Ptolémée en Occident, IV e-XVIe siècle, Turnhout : Brepols, 2009.

3. Voir Laurent PINON, Antonella ROMANO, Hélène VÉRIN, « Renaissance des savoirs scientifiques et techniques dans l'Europe de la Renaissance ? Une introduction », introduction au numéro spécial de la Nouvelle Revue du Seizième Siècle, 20, 2002-1.

4. Lucien GALLoIs, Les géographes allemands de la Renaissance, Paris : E. Leroux, 1890.

5. Gerald STRAUSS, Sixteenth-Century Germany. Its Topography and Topographers, Madison, 1959.

6. Voir notamment Wilhelm MAURER, « Melanchton und die Naturwissenschaften seiner Zeit » in : Archiv für Kulturgeschichte, 44, 1962, p.199-266; Manfred BÜTTNER (dir.), Wandlungen im geographischen Denken von Aristoteles bis Kant, Paderborn: Ferdinand Schöning, 1982; Sachiko KusukawA, The Transformation of Natural Philosophy. The Case of Philipp Melanchthon, Cambridge: Cambridge University Press, 1995; Charlott METHUEN, Kepler's Tübingen. Stimulus to a Theological Mathematics, Ashgate : Adlershot, 1998.

7. Peter Heinz MEURER, « Die Wittenberger Universitätsmatrikel als kartogeschichtliche Quelle» in : Heinz P. BROGIATO et Hans-Martin closs (dir.), Geographie und ihre Didaktik. Festschrift für Walter Sperling, 2, Trier : Geographische Gesellschaft, 1992, p. 201-212.

8. Wolfgang REINHARD, « Gegenreformation als Modernisierung ? Prolegomena einer Theorie des konfessionellen Zeitalters» in: Archiv für Reformationsgeschichte, 68, 1977, p. 226-251; Heinz SCHILLING, «Die Konfessionalisierung im Reich. Religiöser und gesellschaftlicher Wandel in Deutschland zwischen 1555 und $1620 »$ in : Historische Zeitschrift, 246, 1988, p. 1-45.

9. Robert MERTON, Science, Technology and Society in Seventeenth Century England, New York, 1970 (1ère édit., 1938).

10. Cette tendance a été largement contestée par ailleurs. Voir notamment Steven SHAPIN, «Understanding the Merton Thesis » in: Isis, 79, 299, 1988, p. 594-605 et Rivka FELDHAY et Yehuda ELKANA (dir.), «After Merton». Protestant and Catholic Science in Seventeenth-Century Europe, numéro spécial de la revue Science in Context, 3, 1989-1.

11. Cette stratégie est celle retenue par Jean-Marc BESSE, qui établit une histoire intellectuelle du savoir géographique en Europe à la Renaissance et étudie successivement la réception de la Géographie de Ptolémée et le développement des cosmographies modernes, la Cosmographie de Sebastian Münster et le Theatrum orbis terrarum d'Abraham Ortelius. Voir Jean-Marc Besse, Les grandeurs de la Terre. Aspects du savoir géographique à la Renaissance, Lyon : ENS Éditions, 2003. 